Several factors might explain the transatlantic differences in the management of febrile seizures: 1) a greater availability and reliance on rectal diazepam for use by parents in the U.K., 2) geographic considerations and the greater distances to hospitals for emergency room treatment of the acute seizure in rural areas of the U.S., 3) the apparent importance of parental anxiety and physician concern for litigation in the U.S. Therapeutic guidelines may be helpful but the decision to treat must be made on an individual basis. When further and more carefully evaluated, perhaps the EEG will prove of value and receive attention in febrile seizure management.

\title{
THE EEG AND FEBRILE SEIZURES
}

The relationship between clinical characteristics and EEG classification in 676 children with febrile seizures was examined at the University Pediatric Clinic, Skopje, Yugoslavia. The initial EEG was classified as paroxysmally abnormal (spike wave complexes, generalized or focal, or spikes) in $22 \%$. Focal seizures and those that lasted more than 15 minutes were more often associated with abnormal EEGs. Multiple seizures in the first 24 hours was not related to risk of specific paroxysmal abnormalities. The leading predictors of initial paroxysmal EEG abnormality were 1) older age at the time of EEG and 2) the number of previous febrile seizures. Motor abnormality before the index seizure and focal seizure had less predictive value. The rate of EEG abnormality in children with no previous seizures was $18 \%$ whereas in those with 4 or more previous seizures it was $63 \%$ (Sofijanov $\mathrm{N}$ et al. Febrile seizures: clinical characteristics and initial EEG. Epilepsia Jan/Feb 1992; 33:52-57). (Reprints: Dr. K. B. Nelson at NIH, Federal Building, Room 700, Bethesda, MD 20892, U.S.A.)

COMMENT. Age at the time of the EEG, the number of seizures experienced and a focal first febrile seizure were all predictive of a paroxysmal EEG abnormality. In children more than 4 years of age abnormal EEGs were found in $51 \%$. Further follow-up of this population based sample should allow correlation of the initial EEG findings and the occurrence of subsequent afebrile seizures and epilepsy. In a questionnaire survey of pediatricians and pediatric neurologists in the U.S.A., the EEG was used in determining the need for long-term phenobarbital prophylactic therapy for febrile seizures by $51 \%$ and $45 \%$, respectively. Paroxysmal EEG abnormalities were more frequent in children 5 years of age and older than in younger age groups, and the incidence of EEG abnormalities in records of patients who developed afebrile seizures was 5 times that observed in children with febrile seizures alone. Paroxysmal tracings were more frequent in patients with prolonged or focal febrile seizures than in patients with short simple seizures (Millichap JG, Clinical EEG. 1991; 22:5-12. Idem, Febrile convulsions, 1968, McMillan, New York). 